Portland State University

PDXScholar

\title{
Examining the Reproducibility and Feasibility of Developing Silicon Dioxide Coated Bismuth Nanoparticles as Potential Imaging Contrast Agents
}

Aishwarya S. Shimpi

Portland State University

Follow this and additional works at: https://pdxscholar.library.pdx.edu/honorstheses Let us know how access to this document benefits you.

\section{Recommended Citation}

Shimpi, Aishwarya S., "Examining the Reproducibility and Feasibility of Developing Silicon Dioxide Coated Bismuth Nanoparticles as Potential Imaging Contrast Agents" (2017). University Honors Theses. Paper 459.

https://doi.org/10.15760/honors.456

This Thesis is brought to you for free and open access. It has been accepted for inclusion in University Honors Theses by an authorized administrator of PDXScholar. Please contact us if we can make this document more accessible: pdxscholar@pdx.edu. 
Examining the reproducibility and feasibility of developing silicon dioxide coated bismuth nanoparticles as potential imaging contrast agents

$$
\text { by }
$$

Aishwarya Shimpi
An undergraduate honors thesis submitted in partial fulfillment of the
requirements for the degree of
Bachelor of Science
in
University Honors
and
Biology

Thesis Adviser

Dr. Andrea Goforth

Portland State University

2017 


\begin{abstract}
The development of novel X-ray contrast agents (XCAs) is being widely investigated. Nanoparticles for use as potential contrast agents is a heavily researched area because nanomaterials are able to generate a higher contrast image relative to a small molecule, have long blood circulation times, and allow for the addition of different functionalities such as a surface coating. Particularly bismuth nanoparticles are being considered as potential X-ray contrast agents because of bismuth's high X-ray opacity and high biological tolerability. Furthermore, an $\mathrm{SiO}_{2}$ shell coating around bismuth nanoparticles can offer benefits in regards to nanoparticle colloidal stability in aqueous solution and in vivo biocompatibility. In this research, an aerobic synthesis of elemental bismuth nanoparticles and subsequent silicon dioxide coating protocol is examined in order to examine the reproducibility and feasibility of developing uniform silicon dioxide coated bismuth nanoparticles. TEM analysis was conducted and the images were analyzed for particle size distribution, shell thickness, and shape. The results of the elemental bismuth preparation trials show reproducibility in producing nanoparticles with narrow polydispersity within a range of $40-80 \mathrm{~nm}$, but do not show reproducibility in producing size consistent bismuth nanoparticles. The average bismuth nanoparticle size was found to be 58.35 $\mathrm{nm} \pm 13.47$. The $\mathrm{SiO}_{2}$ procedure results showed that there was a positive association observed between silicon dioxide shell thickness and 25, 50, and $100 \mu \mathrm{L}$ of Tetraethyl orthosilicate (TEOS) amounts with a limiting effect and that the procedure was effective in coating BiNPs uniformly with small variabilities. Both procedures could be improved and the limitations could be decreased to achieve highly monodisperse $\mathrm{SiO}_{2} \mathrm{BiNPs}$.
\end{abstract}


Cover Page

Abstract

List of Figures and Tables

a. $X$-ray Radiography

b. X-ray Attenuation

c. Contrast Agents

7-8

d. Nanoparticles as Contrast Agents

e. Bismuth Nanoparticles and Synthesis

9-10

f. Use of Silicon-dioxide Shell Coatings and Synthesis

10

2. Experimental Section

11-15

a. Materials

b. Synthetic Preparations

11-13

c. Instrumentation

13-14

d. Analysis Methods

14-15

3. Results and Discussion

a. Analysis of BiNPs Synthesized Using the Aerobic Elemental Bismuth Synthesis preparation

b. Analysis of $\mathrm{SiO}_{2}$ Coated BiNP

22-29

4. Conclusions

30-31

5. Future Work 
$\begin{array}{lll}\text { Figure } 2 & \text { TEM image of the first batch of BiNPs } & 16\end{array}$

Figure $3 \quad$ Particle size distribution for the TEM image from Figure 2 16

$\begin{array}{lll}\text { Figure } 4 & \text { TEM image of the second batch of BiNPs } & 17\end{array}$

$\begin{array}{lll}\text { Figure } 5 & \text { Particle size distribution for the TEM image from Figure } 4 & 17\end{array}$

$\begin{array}{lll}\text { Figure } 6 & \text { TEM image of the third batch of BiNPs } & 18\end{array}$

$\begin{array}{lll}\text { Figure } 7 & \text { Particle size distribution for the TEM image from Figure } 6 & 18\end{array}$

Figure $8 \quad$ TEM image of $\mathrm{SiO}_{2}$ coated BiNPs that used $100 \mu \mathrm{L}$ of TEOS 22

Figure 9 Core nanoparticle diameter distribution of Figure 8 TEM image 22

Figure $10 \quad \mathrm{SiO}_{2}$ shell thickness distribution of Figure 8 TEM image 23

Figure 11 TEM image of $\mathrm{SiO}_{2}$ coated BiNPs that used $50 \mu \mathrm{L}$ of TEOS 23

Figure 12 Core nanoparticle diameter distribution of Figure 11 TEM image 24

Figure $13 \quad \mathrm{SiO}_{2}$ shell thickness distribution of Figure 11 TEM image 24

Figure 14 TEM image of $\mathrm{SiO}_{2}$ coated BiNPs that used $25 \mu \mathrm{L}$ of TEOS 25

Figure 15 Core nanoparticle diameter distribution of Figure 14 TEM image 25

Figure $16 \quad \mathrm{SiO}_{2}$ shell thickness distribution of Figure 14 TEM image 26

Figure $17 \quad$ TEOS amount effect on $\mathrm{SiO}_{2}$ shell coating thickness 27 
Table $1 \quad$ Summary table of BiNPs size distribution

Table 2 Summary table of statistical values for core diameter for all batches

Table 3 Summary table of statistical values for shell thicknesses of $\mathrm{SiO}_{2}$ for all batches 26 


\section{Introduction- Background and Significance}

a. X-ray Radiography-Since being discovered in 1895 , X-rays have become a significant diagnostic tool. ${ }^{1} \mathrm{X}$-rays have allowed medical professionals to obtain an internal noninvasive visualization of the human body, which has allowed for incredible progress in medical imaging and its application. ${ }^{1}$ The $\mathrm{X}$-ray technique has revolutionized medicine and has provided the medical community inexpensive and high resolution images. ${ }^{1} \mathrm{X}$-ray Radiography involves using a contrast agent to show the physiology or metabolism of the patient internally. ${ }^{2}$ An X-ray image is created by giving a short exposure of $\mathrm{X}$-ray radiation to the patient, that is then represented on a film or detector. ${ }^{3}$ Computed Tomography (CT), which integrates different X-ray images, comprises $75 \%$ of the imaging conducted today. ${ }^{1}$ The field of medical imaging is rapidly expanding. More than 80 million CT scans are performed per year and the number continues to grow at approximately $10 \%$ per year, compared to only 3 million CT scans in $1980 .{ }^{4}$ Figure 1.1 displays examples of medical X-ray images.
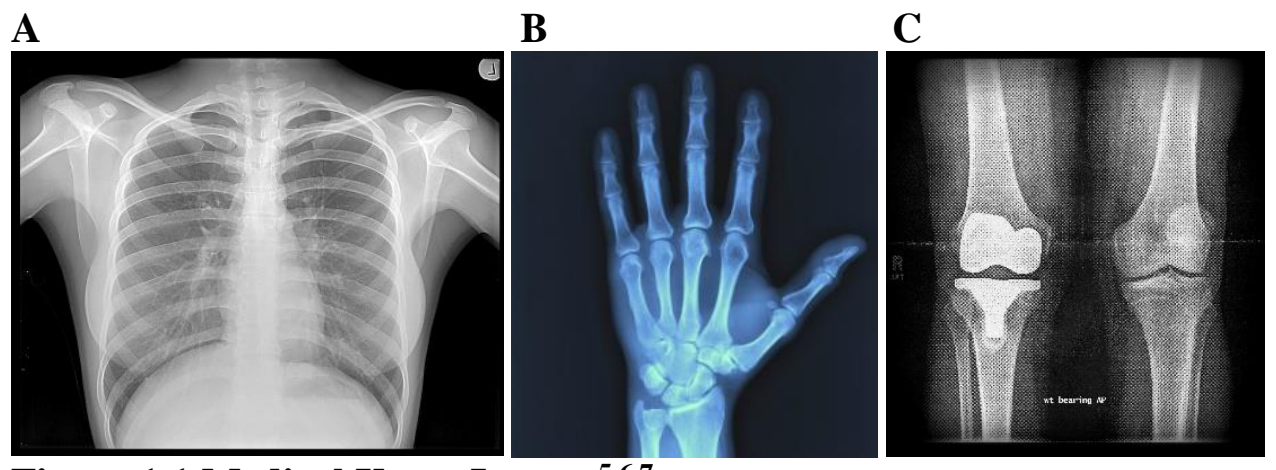

Figure 1.1 Medical X-ray Images ${ }^{5,6,7}$

A) Chest X-ray image B) Hand X-ray Image C) Knee X-ray Image 


\section{b. X-ray Attenuation}

The production of an X-ray image involves attenuation of X-ray radiation. ${ }^{3}$ Attenuation lowers the intensity of an X-ray beam as it passes through a material. ${ }^{8}$ The intensity can be lowered due to several reasons, which include absorption, energy transformation, or deflection of the photons. ${ }^{8}$ The extent of X-ray attenuation depends on the X-ray attenuation coefficient of the attenuating material or substance, $\mu$, which can be calculated by the following formula:

$$
\mu=\frac{\rho Z^{4}}{A E^{3}} \quad \text { Equation } 1
$$

where

$\mu=$ Attenuation Coefficient

$\rho=$ Density of the absorbing material

$\mathrm{Z}=$ Atomic number of atoms in the material

$\mathrm{A}=$ Atomic mass of atoms in the material

$\mathrm{E}=$ energy of the incident X-ray

The attenuation coefficient, $\mu$, thus describes the amount of radiation that can be expected to be attenuated by a given absorbing material. ${ }^{8}$ According to Equation 1 , since $\mathrm{Z}$ is directly proportional to $\mu$, higher atomic number atoms give rise to greater X-ray attenuation. These typically include heavy transition metals, such as bismuth or gold. ${ }^{3}$

\section{c. Contrast Agents}

X-ray contrast agents consist of atoms, molecules, or compounds that are administered internally to a patient for imaging purposes. ${ }^{3}$ The current $\mathrm{X}$-ray contrast agents used most widely are barium sulfate and iodinated molecules. ${ }^{1}$ An estimated 5 million X-ray procedures are conducted with barium sulfate due to it being relatively inexpensive and its ability to pass through a 
patient's GI tract as a mostly undissolved solid. ${ }^{1,3}$ Iodinated molecules have a short circulation time, rapid clearance, and an increased risk of causing thyroid dysfunction but are commonly used for vascular imaging since they are soluble in aqueous media., ${ }^{3,9}$ There are negative side effects associated with traditional contrast agents. Most deaths from contrast media were correlated with renal failure, nephropathy, anaphylaxis or allergic reactions. ${ }^{10}$ This has created a need to replace traditional X-ray contrast media. Specifically contrast media that are less toxic, less costly, and with longer circulation times are among the characteristics suggested. The development of novel X-ray contrast media motivates this research.

\section{d. Nanoparticles as Contrast Agents}

Nanoparticles are typically spherical materials that are between 1 to $100 \mathrm{~nm}$ in size and show potential for imaging purposes. ${ }^{9}$ Nanoparticles as contrast agents are used for several reasons. Nanoparticles can allow generation of a higher contrast image relative to a small molecule, have long blood circulation times, have feasible syntheses, and allow for the addition of different properties such as a shell surface coating. ${ }^{9}$ Nanoparticles contain a large number of X-ray attenuating atoms within a small volume, which would allow for them to be given to a patient internally at a low concentration. ${ }^{11}$ Specifically, elements that have a higher $\mathrm{Z}$ value, such as gold and bismuth, are used as nanoparticle X-ray contrast agents because of their high X-ray opacity. ${ }^{9}$ Gold nanoparticles have been researched as potential contrast agents. However gold nanoparticles have found to be inert and don't readily exit the body. ${ }^{12}$ 


\section{e. Bismuth Nanoparticles}

Bismuth nanoparticles (BiNPs) as potential X-ray contrast agents are the focus of this work.

Bismuth is being considered as a contrast agent for multiple reasons. Bismuth has a high atomic number $(Z=83)$ making it highly $X$-ray opaque and it is found to be biologically well- tolerated. $^{13}$ However, BiNPs are difficult to stabilize in aqueous environments due to their tendency to undergo oxidation and hydrolysis in water. ${ }^{13}$ Although this allows BiNPs to more easily degrade and be cleared through the kidneys, this serves as a challenge in synthesizing BiNPs that can be stabilized long enough for imaging purposes. ${ }^{13}$

Gold nanoparticle synthesis research has helped to provide insight into bismuth nanoparticle synthesis. Therefore, we have developed, an aerobic method for the synthesis of elemental bismuth nanoparticles using bismuth iodide as a precursor. This method was developed to attempt to create more size uniform bismuth nanoparticles with greater reproducibility between replicate batches verses existing reports in the literature. Primarily, two syntheses conducted to form elemental bismuth nanoparticles previously were inspiration in development and optimization of this aerobic method. Wang et al. developed near monodisperse BiNPs between 3-115 $\mathrm{nm}$ in diameter by a simple approach using $\mathrm{Bi}\left[\mathrm{N}\left(\mathrm{SiMe}_{3}\right)_{2}\right]_{3}$ and $\mathrm{Na}\left[\mathrm{N}\left(\mathrm{SiMe}_{3}\right)_{2}\right]$, and a polymer surfactant. ${ }^{14} \mathrm{He}$ et al. also developed highly monodisperse nanoparticles between 5-30 $\mathrm{nm}$ in diameter by using a simple approach that involved reacting the metal chloride precursor with a long chain amine and a strong base to form a metal-amide precursor that decomposed upon heating into the metal nanoparticle. ${ }^{15}$ The synthesis developed by He et al. can be applied to many metals, including bismuth. ${ }^{15}$ Although these syntheses provided useful insight into developing the bismuth nanoparticle synthesis used in this research, these syntheses were performed under strict anaerobic conditions. ${ }^{14,15}$ Conversely, the bismuth 
nanoparticle synthesis developed by our group is able to be conducted under aerobic conditions and also avoids high temperatures or very reactive chemicals. One of the goals of this research is to examine the feasibility of conducting this synthesis in replicate to obtain enough product for contrast agent testing. Ideally, replicate syntheses would result in similar average diameters and standard deviations for the bismuth nanoparticle populations from different batches.

\section{f. Use of Silicon dioxide Shell Coating}

The use of core shell silicon dioxide coated nanoparticles has shown to serve multiple benefits in regards to stability and biological transport of nanoparticles. Additionally, a previous study

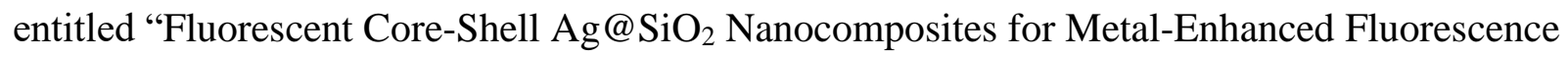
and Single Nanoparticle Sensing Platforms" used silver core nanoparticles with $\mathrm{SiO}_{2}$ shell coating and found that the silica layer can offer "robustness, chemical inertness, and the versatility needed for the conjugation of biomolecules or fluorophores," as well as protect the core and allow for metal enhanced fluorescence. ${ }^{16}$ Based on the results of the study, metal nanoparticles with core shell architecture may be able to yield increase enhancement of fluorescence signal and increase in particle detectability relative to hollow fluorescent nanobubbles (produced by dissolving the metal core by cyanide of the metal-SiO 2 species). ${ }^{15}$ The study specifically used a silver metal species. ${ }^{16}$ Additionally, the $\mathrm{SiO}_{2}$ may aid in the stabilization of storage for BiNPs. Since $\mathrm{SiO}_{2}$ is hydrolyzed in the body and is cleared through the kidneys, this could possibly stabilize bismuth against oxidation for the duration of imaging and allow for clearance after. Hence the benefits of a silicon dioxide coating are being considered for the bismuth core nanoparticles in this research. 


\section{Experimental Section}

Materials, Synthetic Preparations, Instrumentation, and Analysis Methods:

a. Materials -Bismuth (III) Iodide (99.999\%, Strem), di-isopropyl benzene (98\%, Acros), 1hexadecylamine (90\%, Technical Grade, Alfa Aesar), poly(1-vinylpyrrolidone)-graft-(1triacontene) (Sigma-Aldrich), hexane (ACS grade, Fisher), electrophoretically pure $\mathrm{H}_{2} \mathrm{O}$ (to 18 $\mathrm{M} \Omega \cdot \mathrm{cm}$ ), ethanol (ACS grade, Fisher), isopropanol (99\%, Fisher), 30\% ammonium hydroxide, and tetraethoxysilane were purchased and used as received.

\section{b. Synthetic Preparation}

\section{Elemental Bismuth Nanoparticle Synthetic Preparation:}

$300 \mathrm{mg}$ of bismuth iodide was weighed on a laboratory balance and added to a $100 \mathrm{~mL}$ one-neck, round flask. Then $4 \mathrm{~mL}$ of di-isopropylbenzene was added as 4 aliquots to the mixture using a 5 $\mathrm{mL}$ pipette (for a total of $16 \mathrm{~mL}$ of di-isopropylbenzene). This mixture was then stirred with a magnetic stir bar and placed on a hot plate. The temperature was then increased to $180^{\circ} \mathrm{C}$ and the reaction was held at this temperature for approximately 10 minutes. The reaction duration was measured with a stopwatch. A change in the solution color from colorless to orange was observed while heating the mixture to $180^{\circ} \mathrm{C}$. After 10 minutes, the heat was lowered to $100^{\circ} \mathrm{C}$ using a stream of cool air. A dryer is used to cool the mixture. A purple gas $\left(\mathrm{I}_{2}\right.$ gas) was observed to be released from the mixture during the evaporation or cooling process of the mixture. Subsequently, $345 \mathrm{mg}$ of poly(1-vinylpyrrolidone)-graft-(1-triacontene) was weighed and added to the flask. Then, $3.75 \mathrm{~g}$ of hexadecylamine was also weighed and added to the flask. This mixture was kept on the hot plate for approximately 1 hour at $100^{\circ} \mathrm{C}$. During this 1 hour, an intensifying orange color solution was observed in the solution, indicative of the formation of a soluble precursor. Halfway through this 1 hour period, a cap was placed on the flask to stopper it. 
After 1 hour, the heat was again increased to $180^{\circ} \mathrm{C}$. The time was monitored by a stopwatch for 10 minutes. At $180^{\circ} \mathrm{C}$, the mixture began to change color from orange to dark black, which indicated nucleation and growth of BiNPs. After 10 minutes, the reaction was quenched by pouring the solution into a beaker containing electrophoretically pure $\mathrm{H}_{2} \mathrm{O}$ (to $18 \mathrm{M} \Omega \cdot \mathrm{cm}$ ) at room temperature, terminates the growth of BiNPs.

\section{Bismuth Nanoparticle Isolation:}

Centrifugation was used to separate and isolate the bismuth nanoparticles that were synthesized. To accomplish this, $50 \mathrm{~mL}$ of hexane was added to the BiNP solution. The mixture was stirred with a magnetic stir bar. This mixture was then placed in a sonic bath for a few minutes to break up any large aggregates. The mixture was then equally divided into 2 centrifuge tubes. These tubes were then placed into the centrifuge for 10 minutes at a speed of 4.4 RPM.

After 10 minutes, the liquid in the tubes was decanted into a beaker. Then, $25 \mathrm{~mL}$ of hexane and $15 \mathrm{~mL}$ of ethanol were added to both tubes. The tubes were then sonicated for a few minutes. The tubes were then placed back into the centrifuge for 10 minutes at a speed of 4.4 RPM. These steps were then repeated at least twice or until the supernatant is colorless. The bismuth nanoparticles are resuspended in toluene for TEM analysis and subsequently into isopropyl alcohol for shell coating.

\section{Silicon dioxide Coating of the Bismuth Nanoparticles:}

To apply a silicon dioxide coating to the BiNPs, $1 \mathrm{~mL}$ of the synthesized BiNPs as a suspension in isopropyl alcohol was used and added to a 500-mL round bottom flask and stirred with a magnetic stir bar. A pipette was used to measure the $1 \mathrm{~mL}$ of BiNPs (unknown concentration). Then, $250 \mathrm{~mL}$ of isopropanol and $25 \mathrm{~mL}$ of electrophoretically pure $\mathrm{H}_{2} \mathrm{O}$ (to $18 \mathrm{M} \Omega \cdot \mathrm{cm}$ ) at room temperature were added to the flask. A graduated cylinder was used to measure these amounts. 
Subsequently, $4 \mathrm{~mL}$ of $30 \%$ ammonium hydroxide was added to this mixture using a pipette. A variable amount of TEOS (tetraethoxysilane) was then added to the mixture.

This silicon dioxide coating was performed a total of three times on three different samples of BiNPs from the same batch. Each of the three times the silicon dioxide coating procedure was conducted, the TEOS amounts were varied. The TEOS amounts used were $25 \mu \mathrm{L}$, $50 \mu \mathrm{L}$, and $100 \mu \mathrm{L}$. In each case, the TEOS was added $25 \mu \mathrm{L}$ at a time with 5 minutes between the addition of the aliquots (for the $50 \mu \mathrm{L}$ and $100 \mu \mathrm{L}$ trials). After the addition of TEOS, the reaction mixtures were stirred for an additional 15 minutes. The reaction mixtures were then placed in a laboratory freezer $\left(0^{\circ} \mathrm{C}\right)$ for approximately 2 hours. At the end of the 2 hours, the solutions were colorless with a grey hue. Subsequently, the flasks were then placed in a sonic bath for a few minutes to mix the solutions. The solutions were then centrifuged to isolate the silicon dioxide coated bismuth nanoparticles. The $\mathrm{SiO}_{2} \mathrm{BiNP}_{\text {were immersed in ethanol for TEM }}$ analysis, but were not used otherwise for further study.

\section{c. Instrumentation}

\section{TEM analysis:}

Transmission electron microscopy (TEM) provides insight into nanoparticle structure and shape by transmitting a beam of electrons that interact with the nanoparticles as the beam moves through them. ${ }^{17}$ This interaction forms an image that can be magnified to view the nanoparticles more clearly. ${ }^{17}$ The BiNPs and the subsequent silicon dioxide coated BiNPs are assessed using transmission electron microscopy to obtain imaging of the nanoparticles for insight into the size, shape, and distribution. The following is the preparation needed for TEM analysis of the nanoparticle samples. A filter paper is placed on a watch glass. The TEM grid is carefully 
removed from the TEM grid storage box using forceps. The TEM grid is placed on the filter paper with the carbon film side facing up. $4 \mu \mathrm{L}$ of the nanoparticle sample is drawn into a micropipette. 1-2 drops of the nanoparticle sample are placed exactly on the TEM grid. This is then allowed to dry outside for approximately 5 minutes and in the oven for approximately 10 minutes. After the drying process, the TEM grid is placed in the TEM grid storage box using forceps for TEM analysis. This marks the end of the preparation process of the sample for TEM analysis. The nanoparticle imaging was conducted on a Technai F20 TEM operating at $4500 \mathrm{eV}$.

\section{d. Analysis Methods:}

\section{Image J:}

Image $\mathbf{J}$ software was used to find the size and size distribution for the BiNPs without shells and to find the shell thickness distribution for the BiNPs with $\mathrm{SiO}_{2}$. For finding the size and size distribution of the $\mathrm{BiNPs}$ without $\mathrm{SiO}_{2}$ coating, the software program was used to discern the nanoparticles from the background by considering image contrast in defining of particle boundaries. The scale was appropriately set to measure the diameter of these individual nanoparticles by the software program before the determination of individual nanoparticle boundaries. For manual measurements, individual particles were selected by eye (at random) and their boundaries identified. However, it was difficult for the software program to recognize overlapping nanoparticles, and most of those were left out of the sample size statistics, by deleting them manually from the data set. Also, the background was sometimes considered by the program to be small nanoparticles; these data points also were selectively eliminated (manually deleted). These were limitations in analyzing the nanoparticle size distribution that may have affected the mean nanoparticle diameter. Furthermore, $\mathrm{SiO}_{2}$ shell coating thickness 
was also difficult to determine with the software program, since it was difficult for the software to correctly distinguish the location of the core shell interface. Hence these measurements needed to be taken individual by hand. Then for better accuracy, a scale was set, and each individual nanoparticle core diameter was measured by drawing a line through the center of the nanoparticle and measuring its length against the scale. The shell thicknesses were measured the same way. This allowed the experimenter to more accurately distinguish between the shell and the core nanoparticle. However, this method also introduced error in the measurements, that were due to the perception of the experimenter. Thus, neither method can be considered exactly accurate, but rather considered to be approximations that allow relative comparisons in the samples against each other. 


\section{Results and Discussion}

a. Analysis of BiNPs created using the elemental bismuth synthesis preparation

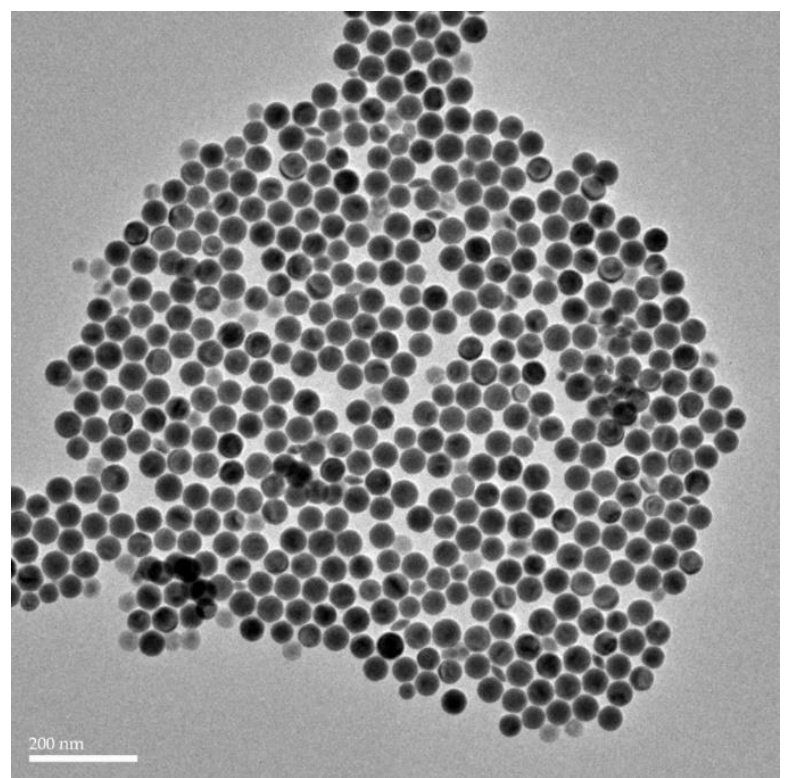

Figure 2: TEM image of the first batch of BiNPs created using the elemental bismuth nanoparticle synthesis preparation. The scale bar is $200 \mathrm{~nm}$.

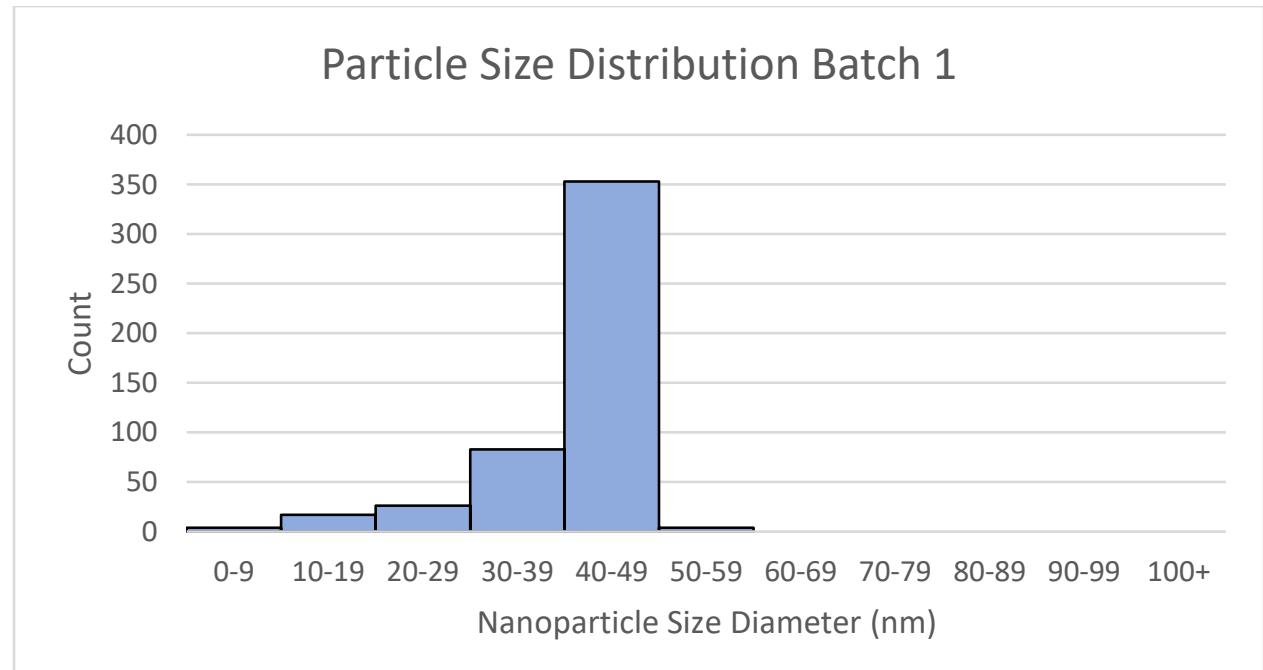

Figure 3: Particle size distribution for the TEM image from Figure 2. The graph displays the number of nanoparticles in each range of nanoparticle size diameters in nanometers $(\mathrm{N}=487)$. 


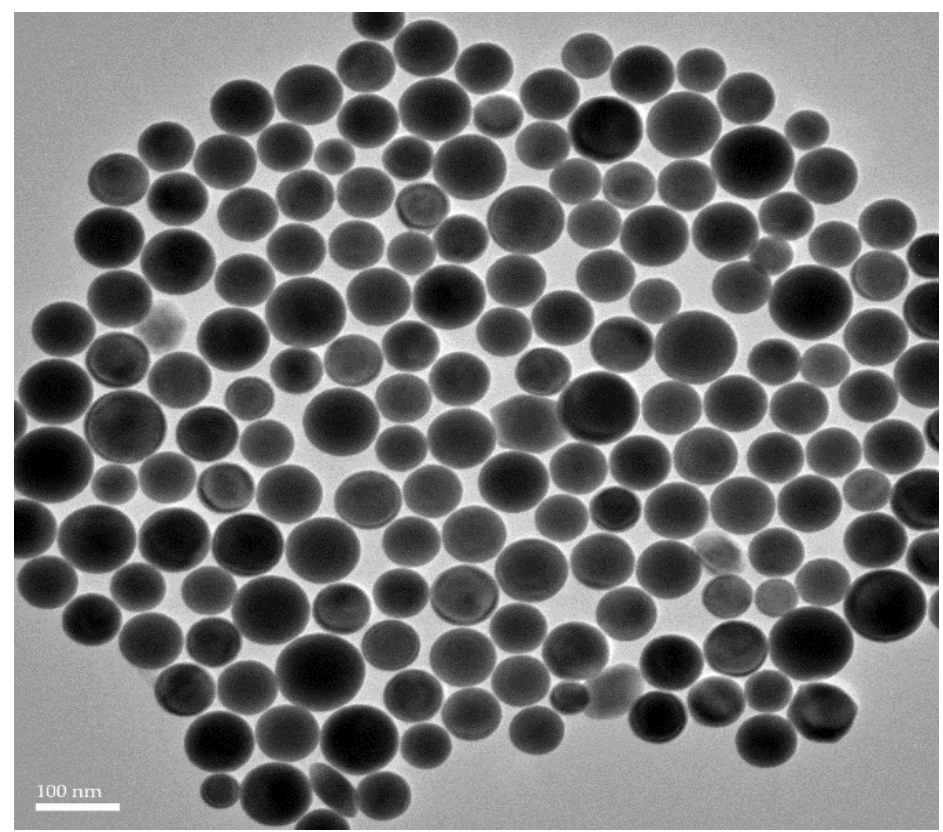

Figure 4: TEM image of the second batch of BiNPs created using the elemental bismuth nanoparticle synthesis preparation. The scale bar is $100 \mathrm{~nm}$.

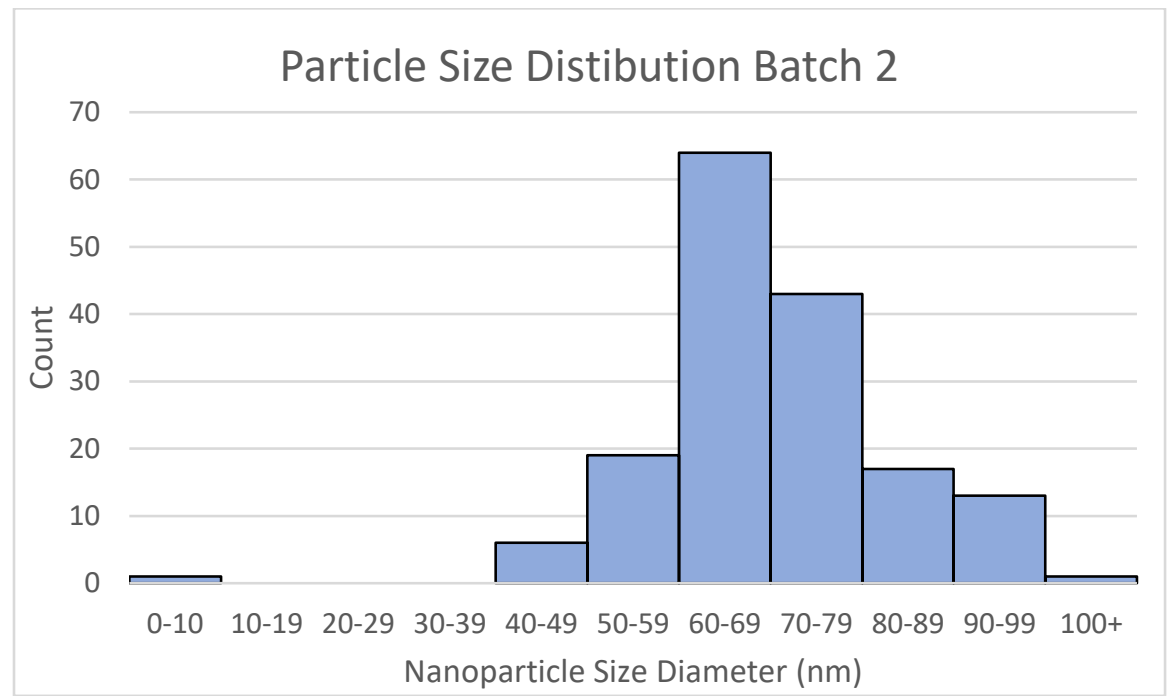

Figure 5: Particle size distribution for the TEM image from Figure 4. The graph displays the number of nanoparticles in each range of nanoparticle size diameters in nanometers $(\mathrm{N}=164)$. 


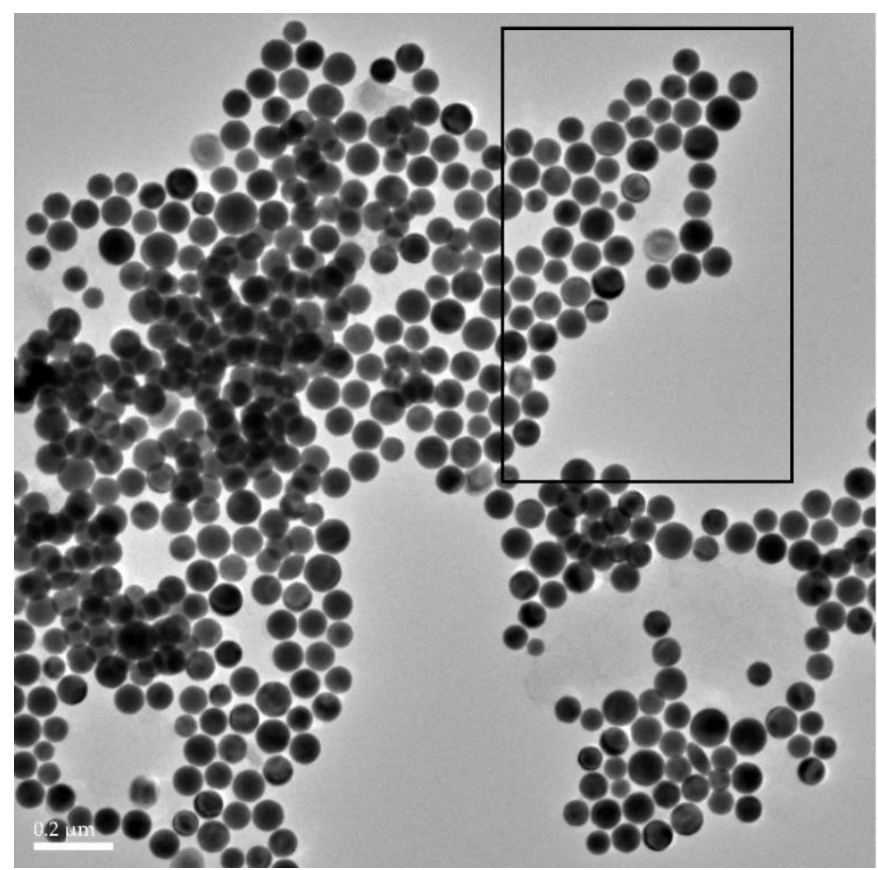

Figure 6: TEM image of the third batch of BiNPs created using the elemental bismuth nanoparticle synthesis preparation. The box shows the sample of nanoparticles used for data analysis. Scale bar is $0.2 \mu \mathrm{m}$.

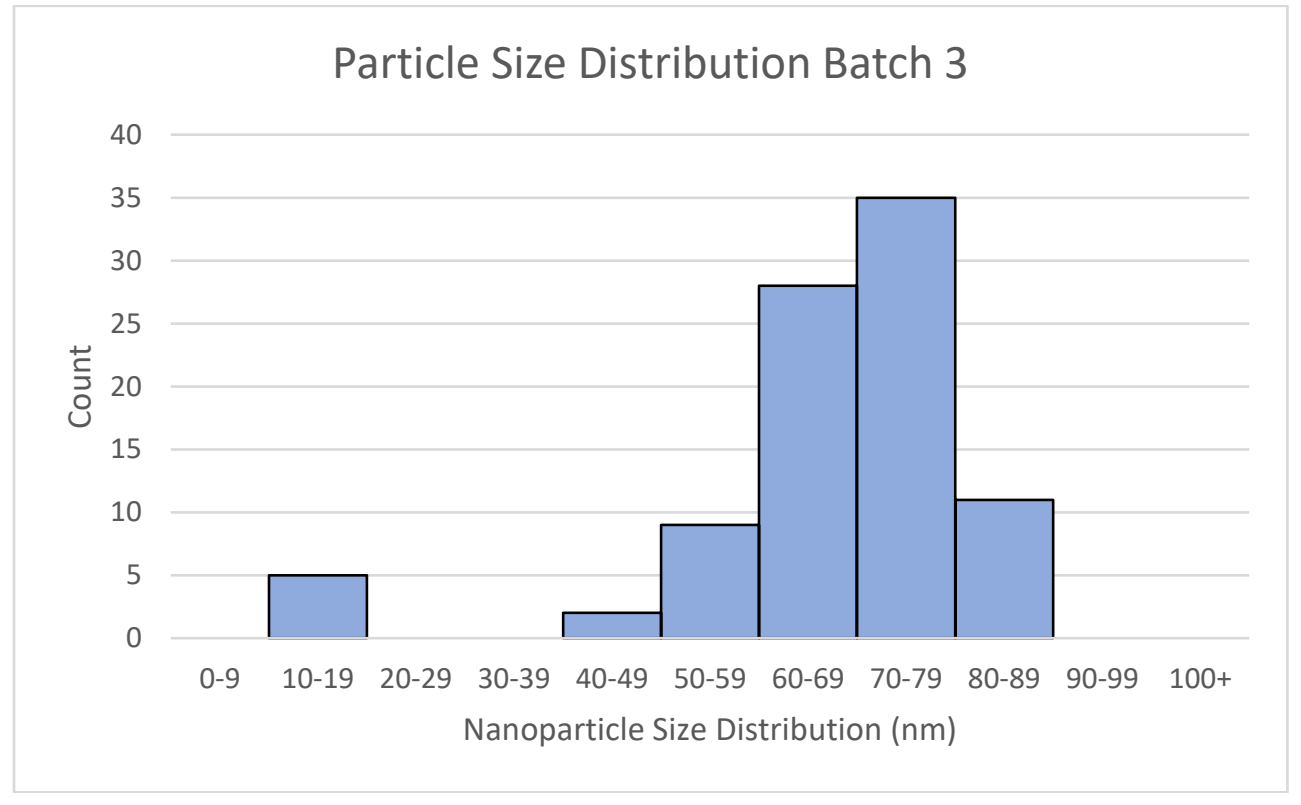

Figure 7: Particle size distribution for the TEM image from Figure 6. The graph displays the number of nanoparticles in each range of nanoparticle size diameters in nanometers $(\mathrm{N}=90)$. 
Table 1: Summary Table of BiNPs size distribution. Includes the mean, median, and standard deviation (S.D.) of nanoparticles (Mean $58.35 \mathrm{~nm} \pm 13.47$ ). The coefficient of variation is calculated by dividing the standard deviation by the mean.

\begin{tabular}{|l|r|r|r|}
\hline & \multicolumn{1}{|l|}{$\begin{array}{l}\text { Mean } \\
(\mathrm{nm})\end{array}$} & $\begin{array}{l}\text { S.D. } \\
(\mathrm{nm})\end{array}$ & $\begin{array}{l}\text { Coefficient of } \\
\text { Variation (\%) }\end{array}$ \\
\hline Batch 1 & 39.37 & 7.71 & 19.6 \\
\hline Batch 2 & 69.24 & 12.53 & 18.0 \\
\hline Batch 3 & 66.45 & 16.00 & 24.0 \\
\hline Mean of Batches & 58.35 & 13.47 & 23.0 \\
\hline
\end{tabular}

Shape and appearance of BiNPs: Nanoparticles should be of a very similar shape for the synthesis to be reproducible at forming uniform BiNPs in terms of appearance. Comparing the shape of the nanoparticles from the TEM images in Figures 2, 4, and 6, it is seen that the shape of the nanoparticles is spherical in all the trials. This indicates that the BiNP synthesis is highly reproducible at producing uniform results in terms of shape.

Size distribution of BiNPs: Three different trials were done to synthesize the BiNPs using the synthetic preparation described in the experimental section. Figures 3, 5, and 7 show the size distribution of bismuth nanoparticles for trials 1, 2, and 3 respectively. Figure 3 shows a majority of the nanoparticles concentrated between $40-50 \mathrm{~nm}$. Figure 5 displays a graph similar to a standard normal distribution curve with a majority of the nanoparticles between 60-80 nm. Figure 7 also displays a graph similar to a normal distribution curve with a majority of the nanoparticles between $60-80 \mathrm{~nm}$ as well. These results show that the second and third trial results for the mean nanoparticle size distributions were much closer and more consistent in comparison to the first trial. The mean nanoparticle sizes were found to be $39.36 \mathrm{~nm} \pm 7.71,69.25 \mathrm{~nm} \pm$ 12.53 and $66.45 \mathrm{~nm} \pm 16.00$ for the first, second, and third trial respectively as seen in table 1 . 
The mean nanoparticle size of all three trials $(58.35 \mathrm{~nm} \pm 13.47)$ was found by calculating the average of the three trials conducted (displayed in table 1). It is also important to note that the average nanoparticle sizes are well below $100 \mathrm{~nm}$ (smaller nanoparticles may be used biologically). The target diameter is $50 \mathrm{~nm}$ however, because this size is ideal for administering an intravenous contrast agent. The mean diameter for all three trials $(58.35 \mathrm{~nm} \pm 13.47)$ is close to $50 \mathrm{~nm}$ and its standard deviation encompasses $50 \mathrm{~nm}$. However, the average nanoparticle diameters of trials 1,2, and 3 are not as close to $50 \mathrm{~nm}$ in comparison to the mean diameter and do not have standard deviations that encompass $50 \mathrm{~nm}$. The majority of the bismuth nanoparticles among all three trials are between $40-80 \mathrm{~nm}$ based on the size distribution graphs (figures 3, 5, and 7). These results indicate that the synthesis is not highly reproducible in achieving exactly $50 \mathrm{~nm}$ bismuth nanoparticles, but is highly reproducible in producing nanoparticles within a specific range between $40-80 \mathrm{~nm}$.

There is some variability within and between each trial conducted. The coefficient of variations are $19.6 \%, 18 \%$, and $24 \%$ for trials 1,2 , and 3 respectively as seen in table 1 . The coefficient of variation for the mean of all three trials is $23 \%$. Overall, the standard deviations and coefficient of variations do show narrow size polydispersity (in this research it is defined as less than $25 \%$ variation) within each trial and between all three trials. The variations are between 19.6-24\%, which are not relatively large in comparison to the small nanoparticle sizes. However, the mean nanoparticle sizes and size distributions for all three trials indicate that producing bismuth nanoparticles of a constant diameter is not reproducible (between and within different batches), despite the limited range in variation. Hence the synthesis is not reproducible in producing nanoparticles of the same size, however there is a high degree of reproducibility in the synthesis to produce nanoparticles that are of a narrow polydispersity. 
As mentioned in the methods used for analysis (chapter 2), there were limitations in using Image J. Using Image $\mathbf{J}$, it was difficult to completely separate overlapping nanoparticles in the TEM images, and those nanoparticles were excluded from the sample thus creating a reduction in sample size. Additionally, an important aspect to note that there is a difference in sample size between the three trials. Using a larger and identical sample size for all trials may allow to decrease the coefficient of variation and standard deviation, which may show a greater degree of reproducibility and uniformity in the size of the nanoparticles. These trials are subject to experimental errors such as timing, measurement of materials, and variations in the instruments (mass scale and hotplate primarily). Hence multiple trials may lower the variations seen in results and show a greater degree of reproducibility of the synthetic bismuth preparation in producing size uniform and monodisperse nanoparticles. Additionally, despite limitations and sources of error, there may be improvements that can be made to the synthesis in order to produce highly monodisperse BiNPs. 
b. Analysis of $\mathrm{SiO}_{2}$ BiNP Core Nanoparticles and Shells

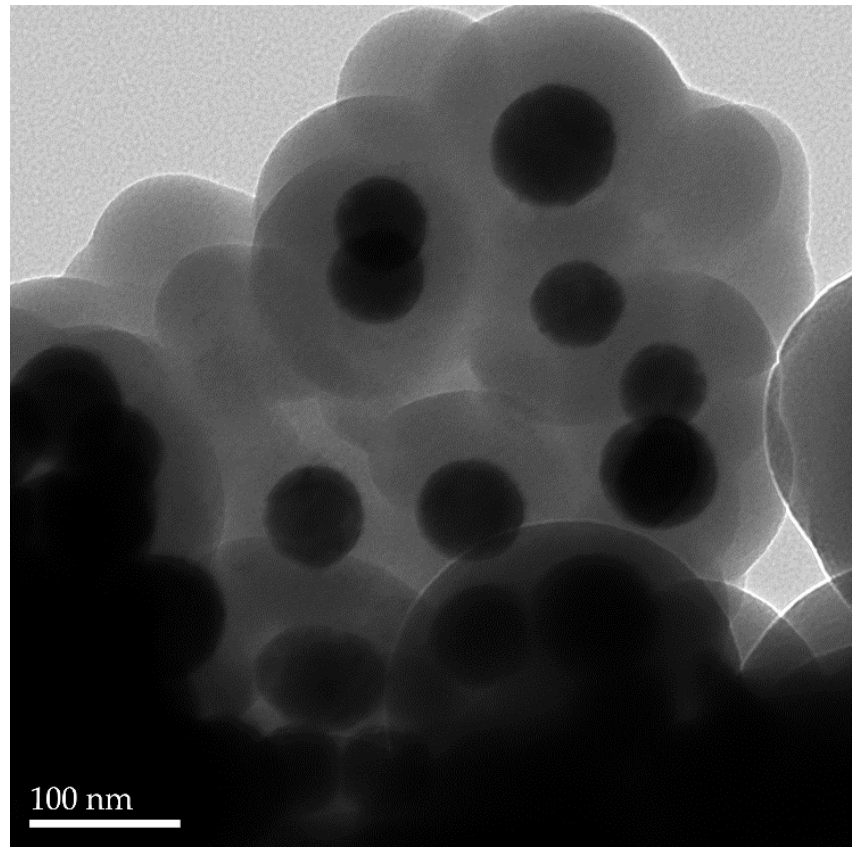

Figure 8: TEM image of $\mathrm{SiO}_{2}$ Coated BiNPs that used $100 \mu \mathrm{L}$ of TEOS. The scale bar is 100 $\mathrm{nm}$.

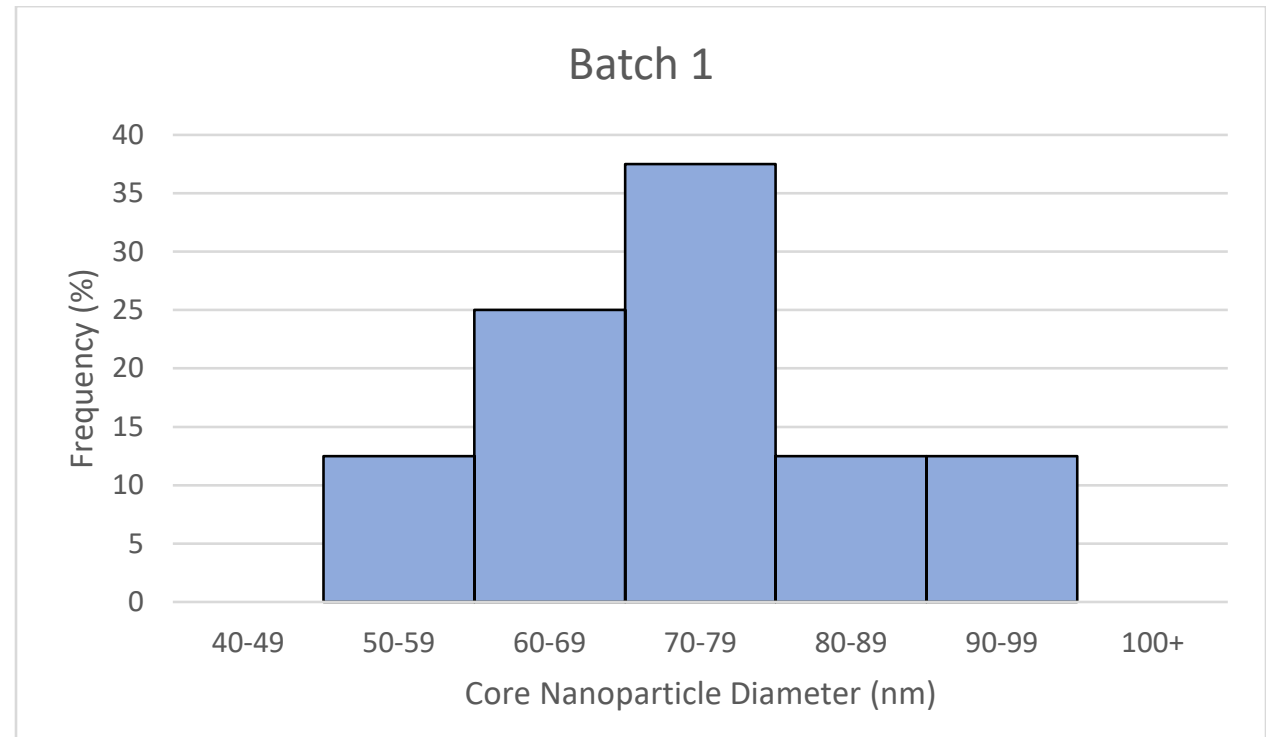

Figure 9: Core nanoparticle diameter distribution from the Figure 8 TEM image. The frequency represents the percent of nanoparticles within each range of core diameter lengths $(\mathrm{N}=8)$. 


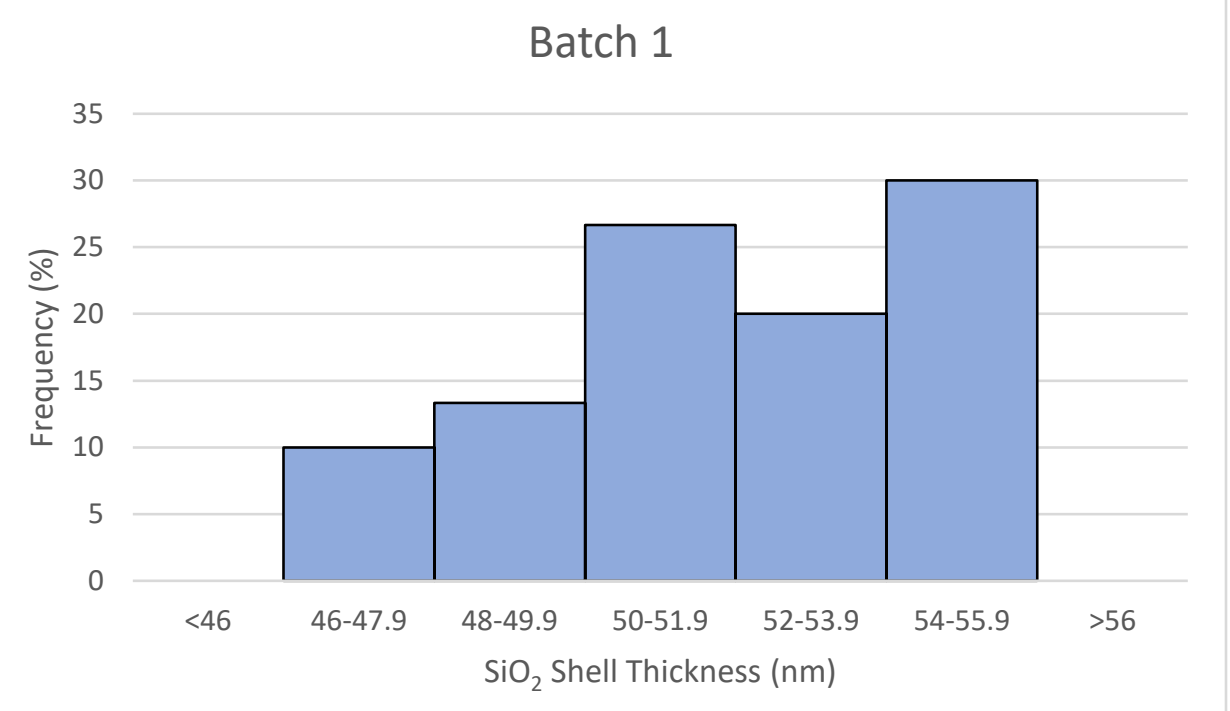

Figure 10: $\mathrm{SiO}_{2}$ Shell thickness distribution from the Figure 8 TEM image $(\mathrm{N}=30)$. The frequency represents the percent of shell thickness measurements within each range of the total sample size. Multiple shell thickness measurements were taken for each nanoparticle in Figure 8, because the shell thicknesses varied around each nanoparticle.

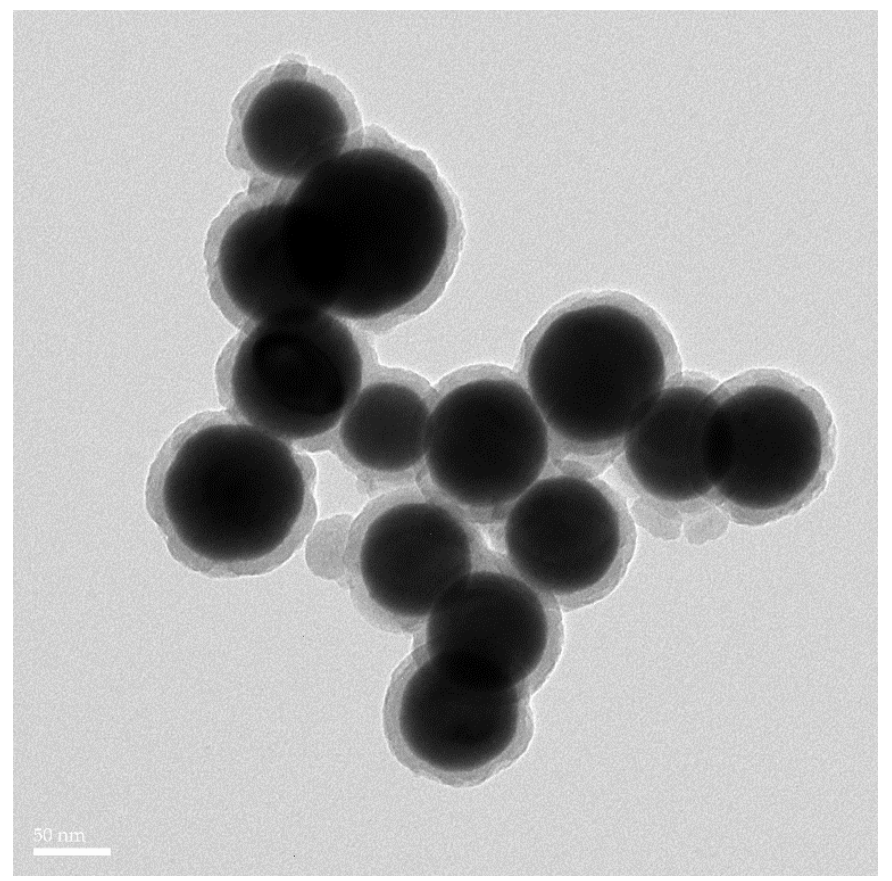

Figure 11: TEM image of $\mathrm{SiO}_{2}$ coated BiNPs that used $50 \mu \mathrm{L}$ of TEOS. Scale bar is $50 \mathrm{~nm}$. 


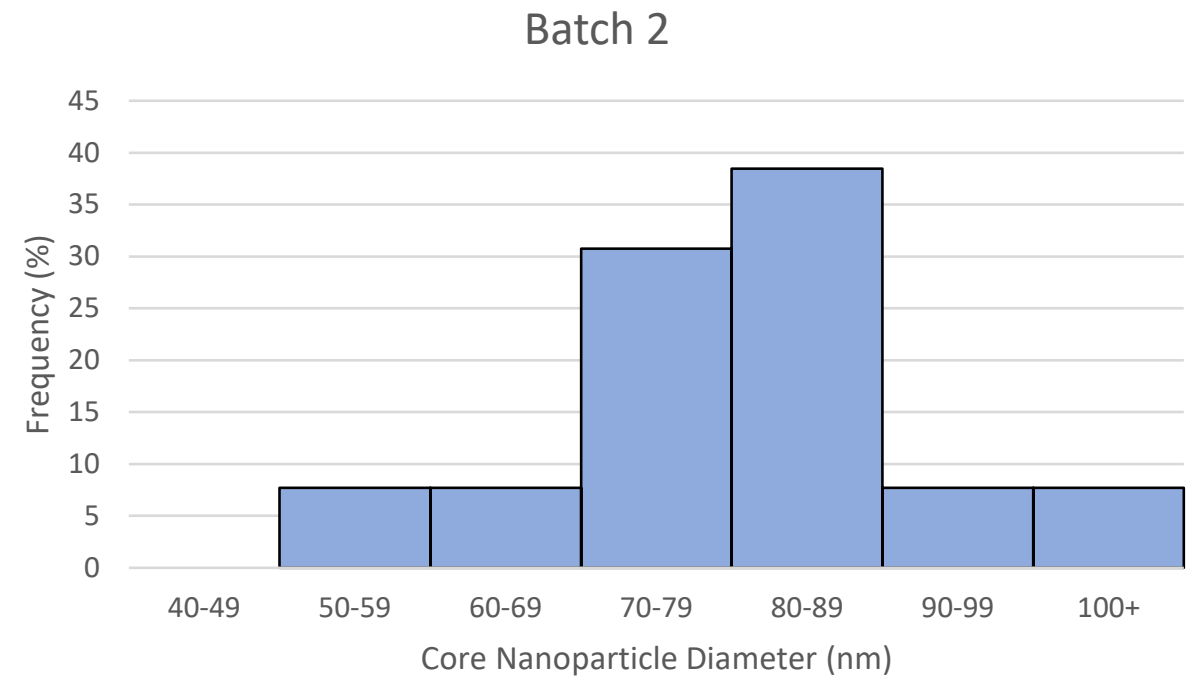

Figure 12: Core nanoparticle diameter distribution from the Figure 11 TEM image. The frequency represents the percent of nanoparticles within each range of core diameter lengths $(\mathrm{N}=14)$.

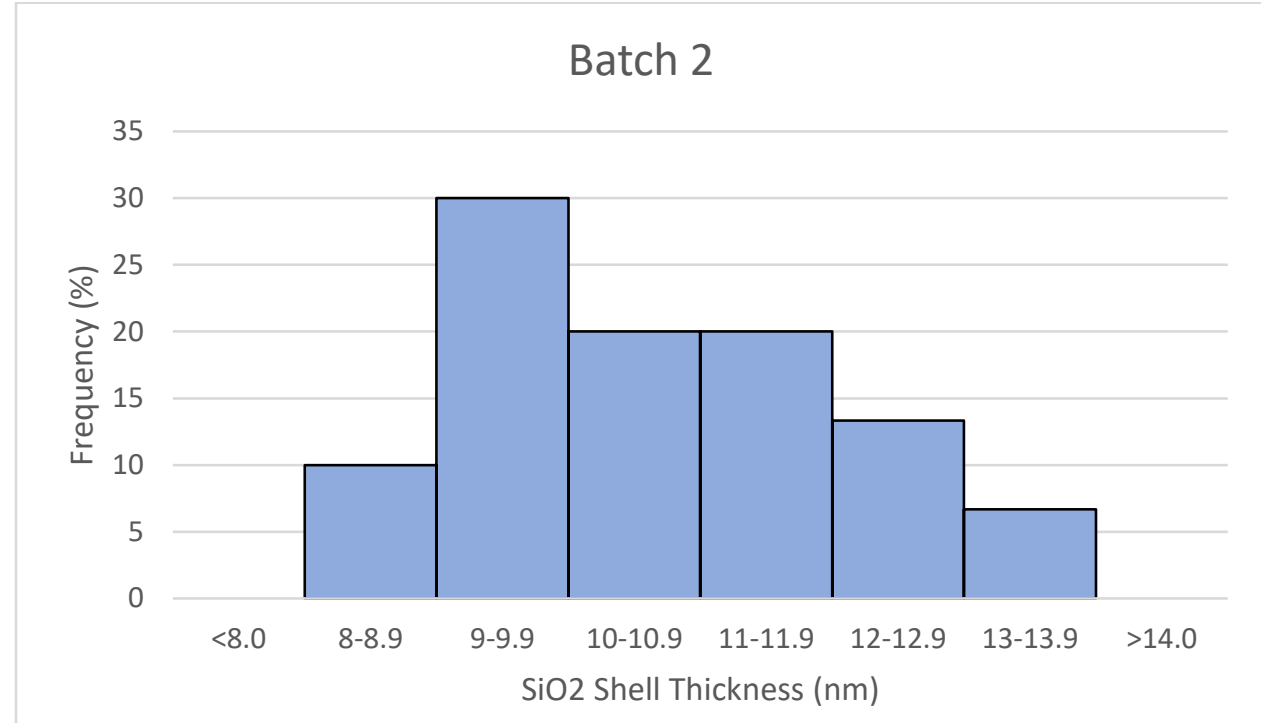

Figure 13: $\mathrm{SiO}_{2}$ Shell Thickness distribution of Figure 11 TEM Image $(\mathrm{N}=30)$. The frequency represents the percent of shell thickness measurements within each range of the total sample size. Multiple shell thickness measurements were taken for each nanoparticle in Figure 11, because the shell thicknesses varied around each nanoparticle. 


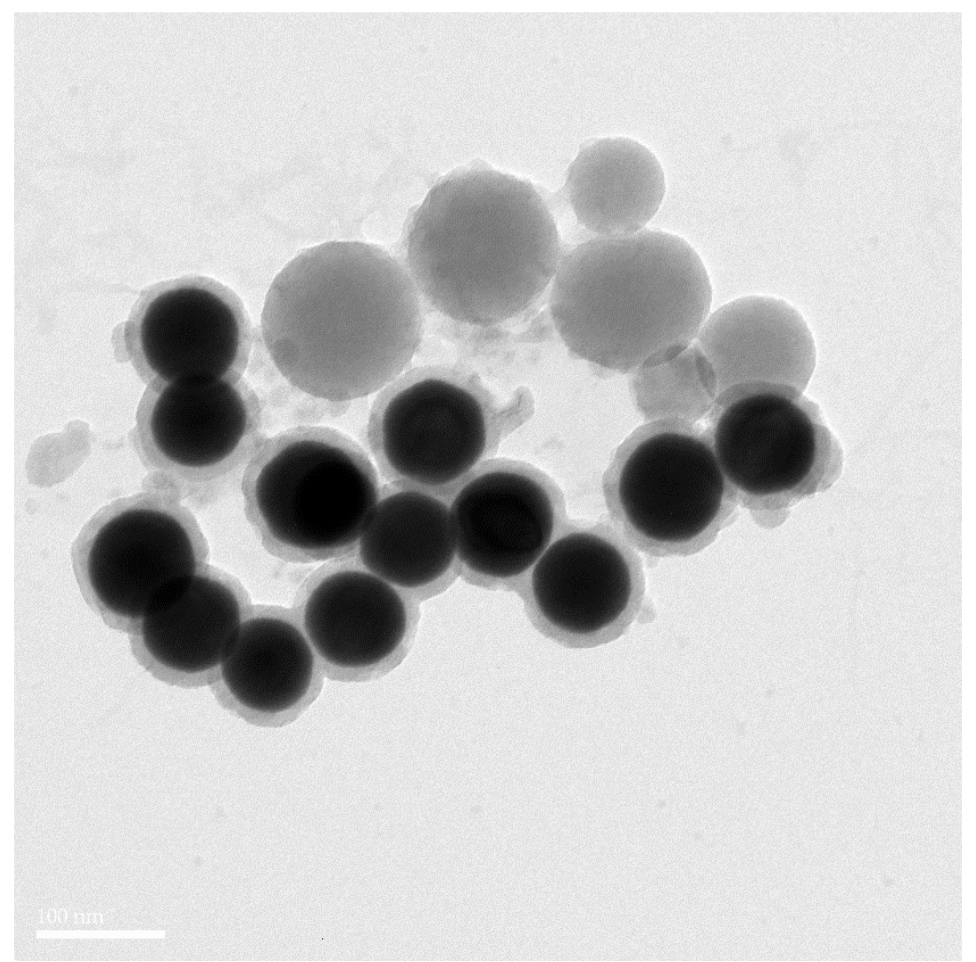

Figure 14: TEM image of $\mathrm{SiO}_{2}$ coated BiNPs that used $25 \mu \mathrm{L}$ of TEOS. The scale bar is 100 $\mathrm{nm}$.

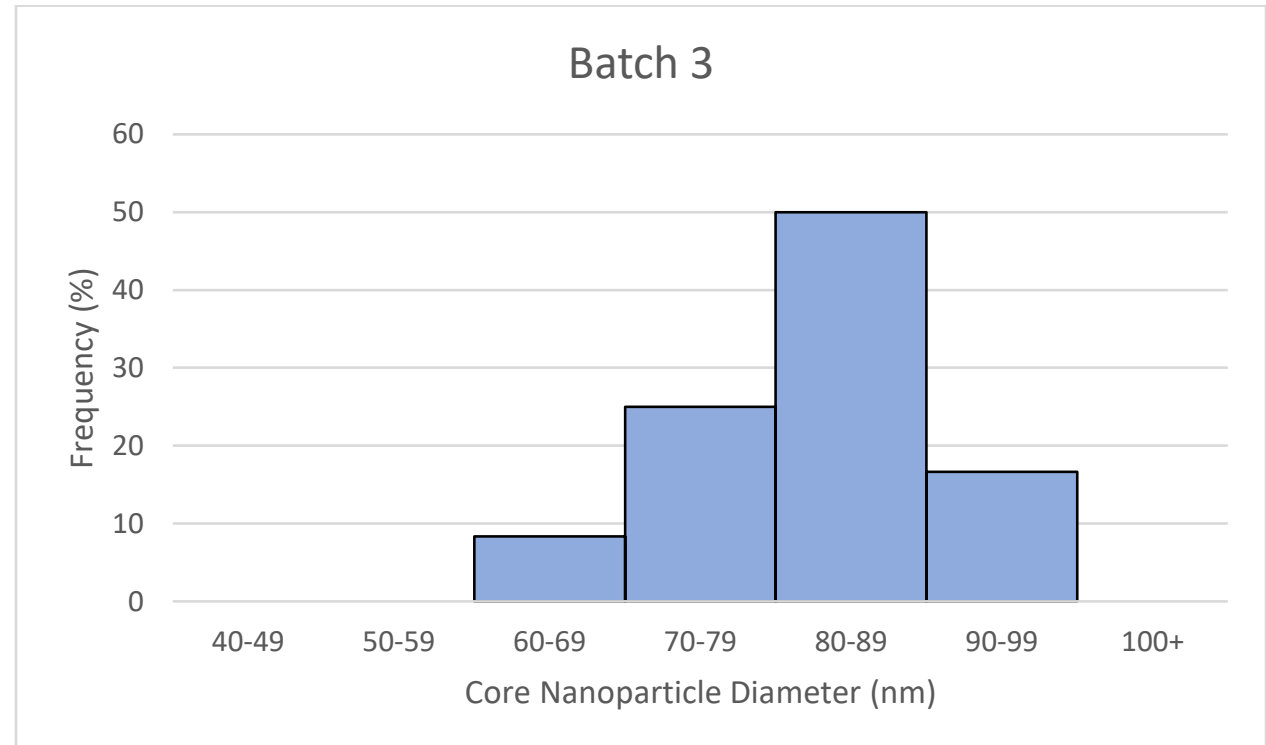

Figure 15: Core nanoparticle diameter distribution from Figure 14 TEM image. The frequency represents the percent of nanoparticles within each range of core diameter lengths $(\mathrm{N}=13)$. 


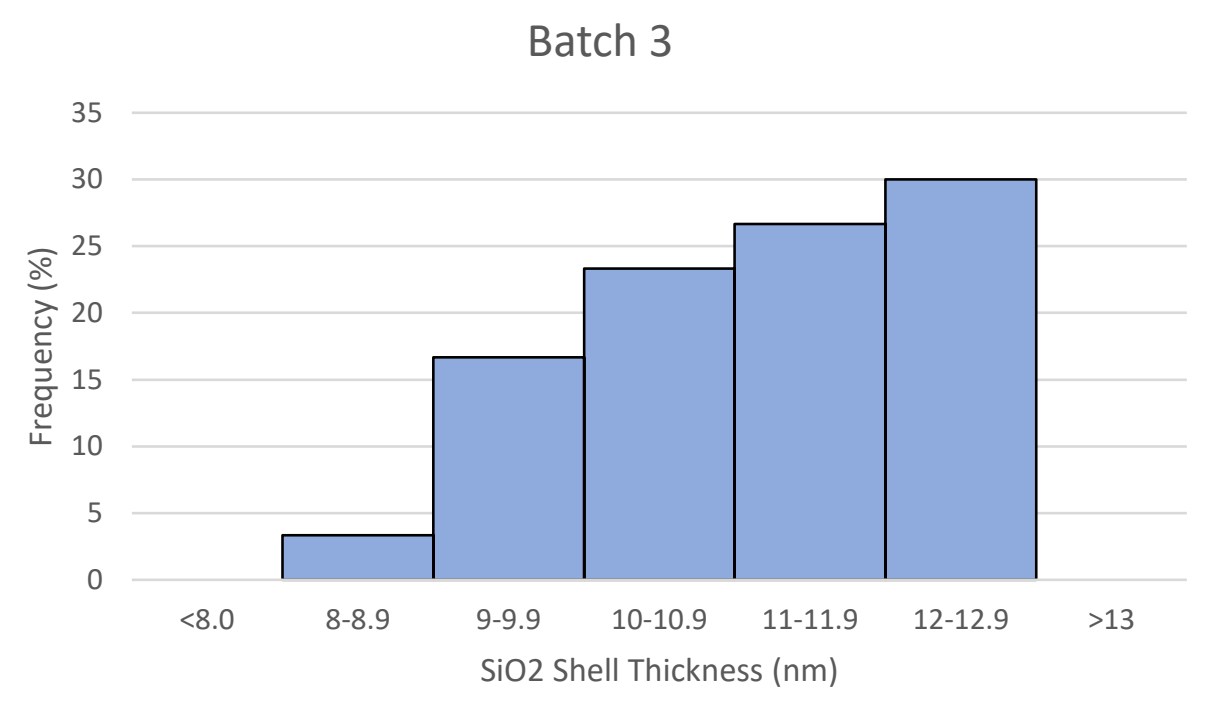

Figure 16: $\mathrm{SiO}_{2}$ shell thickness distribution from Figure 14 TEM Image $(\mathrm{N}=30)$. The frequency represents the percent of shell thickness measurements within each range of the total sample size. Multiple shell thickness measurements were taken for each nanoparticle in Figure 14, because the shell thicknesses varied around each nanoparticle.

Table 2: Core Diameter: Summary table of statistical values for core diameter for all batches. The mean is $78.59 \pm 3.97$ for the core diameter of all batches of nanoparticles. The coefficient of variation is calculated by dividing the standard deviation by the mean.

\begin{tabular}{|l|r|r|r|}
\hline & $\begin{array}{l}\text { Mean } \\
(\mathrm{nm})\end{array}$ & S.D. $(\mathrm{nm})$ & $\begin{array}{l}\text { Coefficient } \\
\text { of Variation } \\
(\%)\end{array}$ \\
\hline Batch 1 & 72.98 & 9.96 & $13.6 \%$ \\
\hline Batch 2 & 81.11 & 12.37 & $15.3 \%$ \\
\hline Batch 3 & 81.67 & 6.16 & $7.5 \%$ \\
\hline Mean of Batches & 78.59 & 3.97 & $5.0 \%$ \\
\hline
\end{tabular}

Table 3: $\mathrm{SiO}_{2}$ Shell Coating: Summary table of statistical values for shell thicknesses for all batches. The coefficient of variation is calculated by dividing the standard deviation by the mean.

\begin{tabular}{|l|r|r|r|}
\hline & Mean (nm) & S.D. (nm) & $\begin{array}{r}\text { Coefficient of } \\
\text { Variation (\%) }\end{array}$ \\
\hline Batch 1 & 51.86 & 2.65 & $5.1 \%$ \\
\hline Batch 2 & 11.24 & 1.24 & $11.0 \%$ \\
\hline Batch 3 & 10.59 & 1.38 & $13.0 \%$ \\
\hline
\end{tabular}




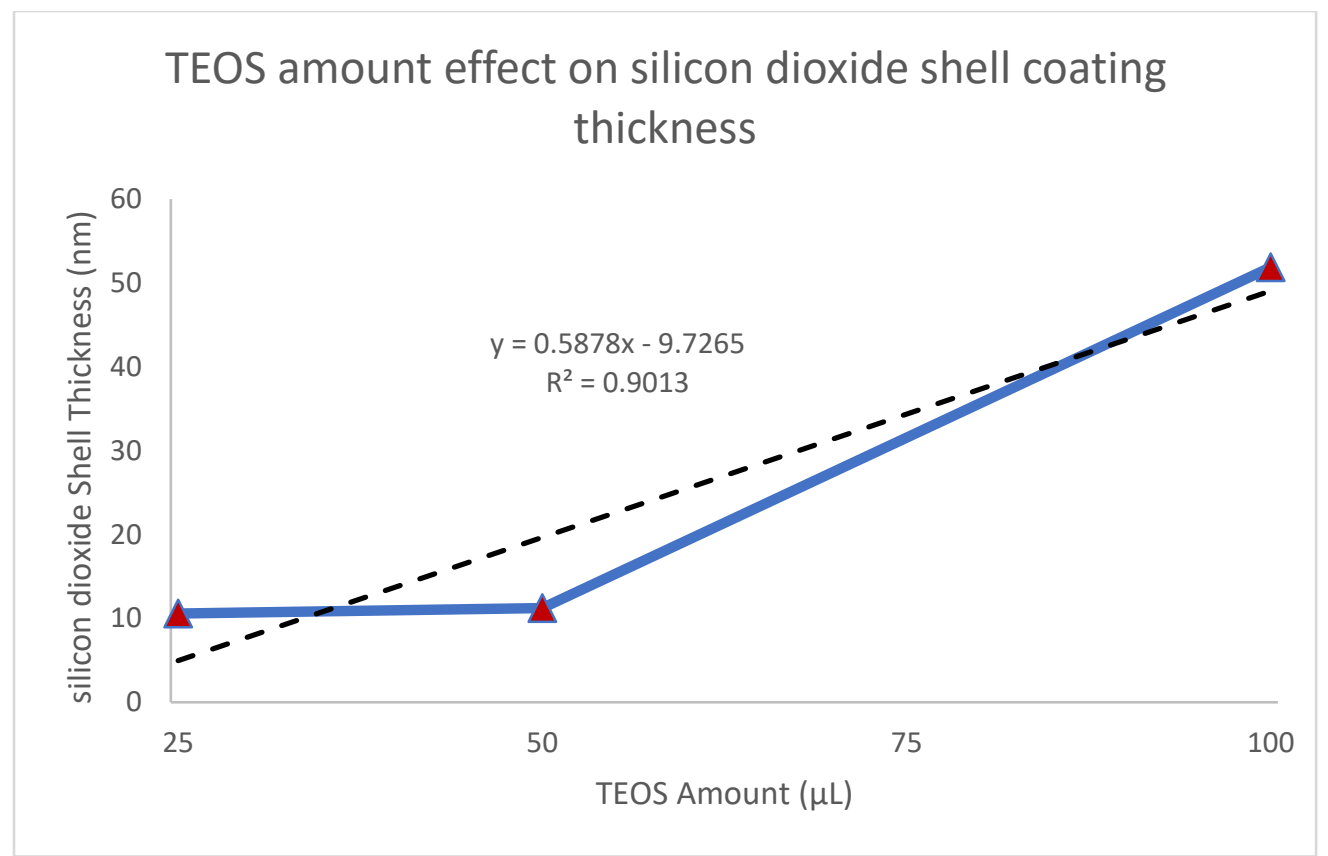

Figure 17: TEOS amount effect on silicon dioxide shell coating thickness

Shape and Appearance: The TEM Images of the $\mathrm{SiO}_{2}$-coated BiNPs are able to clearly show the shell coating, thickness and the core nanoparticle. The shape of the $\mathrm{SiO}_{2}$-coated $\mathrm{BiNPs}$ can be seen as circular. The added $\mathrm{SiO}_{2}$ coating increases the diameter and size of the nanoparticles as a whole. From Figures 8, 11, and 14 it can be seen that the $\mathrm{SiO}_{2}$ shell thickness varies based upon the amount of TEOS added. Figure 17 displays this relationship and shows a positive association for increasing shell thickness with increasing TEOS.

Size Distribution of Core BiNPs: Figures 9, 12, and 15 display the size distribution for the core nanoparticle diameter for batch 1,2 , and 3 respectively. In figures 9,12 , and 15, it can be seen that the core nanoparticle size distributions are primarily centered around $70-90 \mathrm{~nm}$ in diameter. The average core size diameter for all the samples was found to be $78.59 \pm 3.97 \mathrm{~nm}$ (seen in table 2). The coefficient of variability is $5 \%$ for the average core size diameter. From table 2, it can be seen that the standard deviations and the coefficient of variations are both low and 
between $5-15.3 \%$. It is interesting to note that this average core diameter size is higher than the mean BiNP nanoparticle diameter from Table 1 , which is $58.35 \mathrm{~nm} \pm 13.47$. It is challenging to achieve uniform nanoparticle sizes with the bismuth synthesis. The synthesis may need improvements made to it to achieve more consistent core nanoparticle sizes in every batch produced as well as over multiple batches.

$\mathrm{SiO}_{2}$ Shell Thickness: The shell thickness distributions are shown in figures 10, 13, and 16 for batch 1,2, and 3 respectively. Figure 10 shows the shell thickness coating distribution to be between $46-66 \mathrm{~nm}$ for $100 \mu \mathrm{L}$ of TEOS. Figure 13 shows the shell thickness distribution to be between $8-14 \mathrm{~nm}$ for $50 \mu \mathrm{L}$ of TEOS. Figure 16 shows the shell thickness distribution to be between $8-13 \mathrm{~nm}$ for $25 \mu \mathrm{L}$ of TEOS. Table 3 displays the average shell thickness of different batches of BiNPs that used different TEOS amounts. From Table 3, the average shell thicknesses were $51.86 \pm 2.65 \mathrm{~nm}, 11.24 \mathrm{~nm} \pm 1.34$, and $10.59 \pm 1.38 \mathrm{~nm}$ for the $100 \mu \mathrm{L}, 50 \mu \mathrm{L}$, and $25 \mu \mathrm{L}$ of TEOS amounts respectively. These results indicate there is very significant difference in the shell coating thickness of the TEOS amount of $100 \mu \mathrm{L}$ in comparison to the 50 and $25 \mu \mathrm{L}$ amounts. This is indicative of TEOS contributing toward the formation of the $\mathrm{SiO}_{2}$ shell coating. However, there is a very small difference in $\mathrm{SiO}_{2}$ shell thickness between the $50 \mu \mathrm{L}$ and $25 \mu \mathrm{L}$ of TEOS amounts. This relationship is also displayed in figure 17. This could be because the TEOS may have a limiting effect on shell thickness when smaller amounts are used. Only three data trials were used to create this graph, hence more data points may help to further examine this hypothesis and relationship.

From the TEM Image in Figure 8, it can be seen the TEOS is present in excess (with the $100 \mu \mathrm{L}$ of TEOS) and overly coats the $\mathrm{SiO}_{2}$ nanoparticles. Much of the excess TEOS did not 
coat the bismuth nanoparticles. Excessive coating may be unnecessary and may impact the functionality of the BiNPs. It is ideal to have a greater volume of the nanoparticles be composed of bismuth rather than the $\mathrm{SiO}_{2}$, because the heavy metal bismuth allows for greater X-ray attenuation. ${ }^{3,8}$ Therefore, smaller TEOS amounts that sufficiently coat the nanoparticle (without excess shell coating) are ideal.

The coefficient of variability is $5 \%, 11 \%$, and $13 \%$ for batch 1,2 , and 3 respectively. These variabilities are small, particularly for batch 1 which is $100 \mu \mathrm{L}$ of TEOS. The coefficients of variability and low standard deviations show that the silicon dioxide shell coating procedure is able to uniformly coat the bismuth nanoparticles for each TEOS batch amount with small variabilities.

There were limitations in assessing the shell thickness and core diameter. Image $\mathbf{J}$ software was used for the measurements. The shell thickness and core diameter needed to be measured by drawing a line across the shell or core diameter and measuring the length of the line through Image $\mathbf{J}$ once the scale was set. Since there was some slight variability in performing measurements this way, it is important to note that these shell thicknesses and core diameter values are approximations and not exact values. However, an equal number of shell thickness measurements for each TEOS amount were taken, to maintain a constant sample size in order to obtain more accurate thickness distribution graphs for comparison. The silicon dioxide coating method for bismuth nanoparticles may be improved to achieve more size uniform shell thickness distributions. 


\section{Conclusion and Summary}

The goal of this research was to assess the reproducibility and feasibility of the producing $\mathrm{SiO}_{2}$ BiNPs using the synthetic bismuth nanoparticle preparation method and the silicon dioxide shell coating method. This was done through analyzing TEM images of data of $\mathrm{BiNPs}$ and $\mathrm{SiO}_{2}$ BiNPs. The TEM Images were analyzed and assessed to reveal nanoparticle shape and size, size distributions, and shell thickness. The shape, mean size, size distributions, and coefficients of variation of three different trials of the BiNP synthesis were compared and contrasted to assess for reproducibility of the synthesis. The silicon dioxide shell coating method was assessed to examine the effect varying TEOS amounts had on shell thickness. The $\mathrm{SiO}_{2}$ coating procedure was assessed by looking at the morphology, mean shell thickness, shell thickness distribution, and coefficients of variation to determine the uniformity and the degree of variability in shell coating within each batch.

From these results, multiple assertions can be made about the elemental bismuth nanoparticle synthesis in terms of reproducibility. First, the synthetic bismuth nanoparticle preparation method did show a high degree of reproducibility for producing BiNPs of a circular morphology. Second, the BiNP synthesis is not reproducible in producing nanoparticles of the same size within or between different batches. Third, there is a high degree of reproducibility in the synthesis to produce nanoparticles that are of a narrow polydispersity. Fourth, the synthesis is highly reproducible in producing nanoparticles within a specific range of 40-80 nm.

From the results, there are also multiple assertions that can be made about the $\mathrm{SiO}_{2}$ shell coating procedure for BiNPs. First, an increase in TEOS amount was positively correlated with an increase in $\mathrm{SiO}_{2}$ shell thickness. It may be hypothesized that the TEOS can have a limiting effect up to a certain point, where upon changing the amount of TEOS may have little to no 
effect on the shell thickness. Secondly, the low coefficients of variability, low standard deviations, and uniform shell morphology show that the silicon dioxide shell coating procedure is able to uniformly coat the bismuth nanoparticles for each TEOS batch amount with small variabilities.

It is important to assess both procedures for improvements. The BiNP synthetic preparation may be improved to yield highly monodisperse nanoparticles with greater reproducibility. The $\mathrm{SiO}_{2}$ shell coating can also be improved to coat nanoparticles more effectively with even lower variabilities within each batch of different amount of TEOS used. However, it is equally important to note the limitations in sample size, analysis methods, and experimental errors for the results can lead to a greater degree of variation between and within samples for both procedures. It is important to gain more trials and improve experimental and analysis methods to further assess for reproducibility and uniformity within both the BiNP synthesis and the $\mathrm{SiO}_{2}$ coating procedure. 


\section{Future Work}

Future studies need to work on improving the size diameter consistency of nanoparticles within each batch and between batches. Since previous studies have conducted elemental bismuth nanoparticle synthesis under anaerobic conditions, this synthesis provides a novel and facile production of bismuth nanoparticles. Hence, this may help to more easily develop nanoparticles as potential XCAs under atmospheric conditions, while having less stringent experimental conditions. This would make the preparation of such contrast agents much easier. If multiple consistent results between batches are achieved, a possible next step would be to test the stabilization and effectiveness of these nanoparticles in aqueous or biological media.

In terms of the silicon dioxide shell coating, the silicon dioxide shell coating may help to further stabilize the core shell bismuth nanoparticle. Further studies may examine the stability of core shell nanoparticles coated with silicon dioxide in aqueous or biological media. Another further study could also examine the impact on fluorescence of the silicon dioxide shell on bismuth nanoparticles. The increased stability gained by the $\mathrm{SiO}_{2}$ shell coating may help to not only stabilize but increase the fluorescence and particle detectability of the bismuth nanoparticle. This would aid the findings of the $\mathrm{SiO}_{2}$ silver nanoparticle study, and provide more support to synthesize metal nanoparticles with an incorporated $\mathrm{SiO}_{2}$ shell coating in an effort to create a better XCA. 


\section{Acknowledgements}

I would like to express my sincere gratitude to my advisor, Dr. Andrea Goforth for continuously supporting and mentoring me throughout the research and writing process. Her guidance allowed me to learn about nanoparticle chemistry and gain valuable skills and experiences. I would also like to thank Hayden Winter for mentoring and teaching me throughout the research process and providing me with the opportunity to explore more about bismuth nanoparticle synthesis.

Additionally, I want to thank all of the Goforth lab members (Colin, James, Christine, Elena, and Lydia) for helping and supporting me. They have greatly helped me to understand different experimental techniques and answer my questions about the research process. I would also like to thank the Portland State University Honors College and the community of professors and advisors in it, who have guided and helped me on the thesis process. 


\section{References}

[1] Cole, L. E., Ross, R. D., Tilley, J. M., Vargo-Gogola, T., \& Roeder, R. K. (2015). Gold nanoparticles as contrast agents in x-ray imaging and computed tomography. Nanomedicine, $10(2), 321+$.

[2] Li, X.; Anton, N.; Zuber, G.; Vandamme, T. (2014). Contrast agents for preclinical targeted X-ray imaging. Advanced Drug Delivery Reviews, 76, 116-133.

[3] Brown, A. L. (2013). Bismuth Nanoparticles as Medical X-ray Contrast Agents: Synthesis, Characterization and Applications. Ph.D. Thesis. Portland State University: USA.

[4] Brenner, D. J.; Hricak, H. (2010). Radiation Exposure From Medical Imaging Time to Regulate? Jama, 304(2), 208.

[5] Chest X-ray. Digital Image. Bournemouth private clinic. Web. <bournemouthprivateclinic.co.uk>.

[6] Hand X-ray. Digital Image. Ultimate Medical Library. 18 Nov 2014. Web. $<$ ultimatemedical.edu>.

[7] Knee X-ray. Digital Image. California State University Fullerton Risk Management and Environmental Health and Safety. Web. < rmehs.fullerton.edu>.

[8] Mcketty, M. H. (1998). The AAPM/RSNA physics tutorial for residents. X-ray attenuation. RadioGraphics, 18(1), 151-163.

[9] Hahn, M. A., Singh, A. K., Sharma, P., Brown, S. C., \& Moudgil, B. M. (2011). Nanoparticles as contrast agents for in-vivo bioimaging: current status and future perspectives. Analytical and Bioanalytical Chemistry, 399(1), 3-27.

[10] Wysowski, D. K., \& Nourjah, P. (2006). Deaths Attributed to X-Ray Contrast Media on U.S. Death Certificates. American Journal of Roentgenology, 186(3), 613-615.

[11] Cormode, D. P., Naha, P. C. and Fayad, Z. A. (2014), Nanoparticle contrast agents for computed tomography: a focus on micelles. Contrast Media Mol. Imaging, 9: 37-52.

[12] Alkilany, A. M., \& Murphy, C. J. (2010). Toxicity and cellular uptake of gold nanoparticles: what we have learned so far? Journal of Nanoparticle Research, 12(7), 2313-2333. 
[13] Brown, A. L., \& Goforth, A. M. (2012). PH-Dependent Synthesis and Stability of Aqueous, Elemental Bismuth Glyconanoparticle Colloids: Potentially Biocompatible X-ray Contrast Agents. Chemistry of Materials, 24(9), 1599-1605.

[14] Wang, F., Tang, R., Yu, H., Gibbons, P. C., \& Buhro, W. E. (2008). Size- and ShapeControlled Synthesis of Bismuth Nanoparticles. Chemistry of Materials, 20(11), 3656-3662.

[15] He, M., Protesescu, L., Caputo, R., Krumeich, F., \& Kovalenko, M. V. (2015). A General Synthesis Strategy for Monodisperse Metallic and Metalloid Nanoparticles (In, Ga, Bi, Sb, Zn, $\mathrm{Cu}, \mathrm{Sn}$, and Their Alloys) via in Situ Formed Metal Long-Chain Amides. Chemistry of Materials, 27(2), 635-647.

[16] Aslan, K., Wu, M., Lakowicz, J. R., \& Geddes, C. D. (2007). Fluorescent Core-Shell $\mathrm{Ag} @$ SiO2Nanocomposites for Metal-Enhanced Fluorescence and Single Nanoparticle Sensing Platforms. Journal of the American Chemical Society, 129(6), 1524-1525.

[17] Winey, M., Meehl, J. B., O'Toole, E. T., \& Giddings, T. H. (2014). Conventional transmission electron microscopy. Molecular Biology of the Cell, 25(3), 319-323. 